\title{
Orbital exenteration for eyelid skin carcinoma
}

\author{
Ana Silva Guerra $\cdot$ Rui Barbosa • Miguel Choupina • \\ Carlos Pinho $\cdot$ Matilde Ribeiro $\cdot$ Laranja Pontes
}

Received: 1 September 2010 /Accepted: 24 March 2011/Published online: 5 May 2011

(C) Springer-Verlag 2011

\begin{abstract}
Exenteration of the orbit is a disfiguring and destructive procedure; it is generally performed for orbital malignancies and often provides a significant reconstructive challenge. Our purpose was to evaluate the clinical indications for orbital exenteration in a tertiary referral center and to assess the reconstructive options employed. A retrospective nonrandomized analysis was performed, selecting all patients undergoing orbital exenteration over a 5-year period, between January 2005 and January 2010. Patient demographics, tumor characteristics, and reconstructive techniques used were evaluated. Twenty patients with a mean age of 76.5 years underwent total orbital exenteration. Basal cell carcinoma was the main operative indication (45\%), followed by squamous cell carcinoma (15\%). Reconstructive techniques included cover of the raw orbital cavity with a temporal muscular flap in all cases followed with split skin grafting (25\%), bilaterally pedicle $\mathrm{V}-\mathrm{Y}$ advancement flap (10\%) and a fasciocutaneous island flap of the retroauricular region (65\%). Twenty percent of patients had local complications and all were treated in a satisfactory fashion. Eyelid skin tumors remain an important cause of orbital exenteration. Temporal muscle flap is a reliable and stable reconstructive solution after orbital exenteration and additional aid is supplied with skin grafts or local flaps. This technique ensures a good aesthetic outcome and better situation for later complementary treatments and minimal associated donor site morbidity.
\end{abstract}

\footnotetext{
A. Silva Guerra $(\bowtie)$

Plastic Surgery Department, S. José Hospital,

Lisbon, Portugal

e-mail: anacristinacardososilva@gmail.com

R. Barbosa $\cdot$ M. Choupina $\cdot$ C. Pinho $\cdot$ M. Ribeiro $\cdot$ L. Pontes Plastic Surgery Department, Portuguese Oncology Institute, Porto, Portugal
}

Keywords Eyelid cancer - Orbital extentation · Temporalis muscle flap

\section{Introduction}

Orbital exenteration indicates the removal of all the orbital contents and the periosteum of the orbital cavity. In the majority of these, an oncological procedure for the surgical treatment of a malignant tumor with orbital involvement (primary malignant tumors or, more frequently, secondary involvement of the orbit) is performed. Malignancies of the ocular adnexal area are the most prevalent causes of orbital exenteration and they include basal cell carcinoma, squamous cell carcinoma and sebaceous cell carcinoma [1-7]. Other less common tumors include conjunctival malignant melanoma, adenoid cystic carcinoma of the lacrimal gland and uveal melanoma with extrascleral extension. Less commonly, orbital exenteration can be a palliative procedure (pain control) or a therapeutic intervention in aggressive and unresponsive cases of orbital infection (mucormycosis, cellulitis). Exceptionally, orbital exenteration might be indicated for local control of recurrent benign tumors [1].

Traditionally, orbit exenteration can be divided into three types: total, sub-total, and enlarged orbital exenteration as presented by F. Mouriaux et al., and this classification is based on the extent of the resection performed [4]. Therefore, cases that included excision of adjacent bone are defined as extended or enlarged exenteration. When all orbital contents including the globe and periorbita were removed, exenteration was classified as total. Subtotal procedures are those cases in which a partial removal of orbital tissue with sacrifice of the eye was performed. Others like Frezzoti et al. propose another classification which notes the structures preserved [8]. 
Reconstruction after such a disfiguring major deformity results in a very significant challenge. The primary goals of reconstruction are to allow for detection of recurrent disease, restoration of boundaries between the orbit and surrounding cavities, and an acceptable aesthetic outcome [5]. Depending on the tissue remaining, there are several reconstructive options available; these range from a simple skin graft to more complex methods of local flaps up to free tissue transfer [9-12].

The purpose of this study was to review and evaluate the clinical indications for total orbital exenteration in a tertiary referral center, and to assess the reconstructive methods used and their outcome.

\section{Patients and methods}

All exenteration cases were performed at the Portuguese Oncology Institute of Porto (IPO) over a 5 -year period (between January of 2005 and January of 2010). During this period, 223 patients with eyelid skin cancer were referred to our institute. From this population, 20 patients $(9 \%)$ underwent total orbit exenteration. The remaining 203 patients (91\%) were treated by tumor excision with complementary treatment and eyelid reconstruction when indicated. The authors performed a retrospective, nonrandomized analysis of these 20 cases and evaluated patient demographic data, preoperative diagnosis, tumor type, and location. The duration of disease, progression, and prior treatment were assessed.

\section{Results}

Twenty patients ( 12 males and 8 females), with a mean age of 76.5 years (range 46-95 years), underwent total orbital exenteration. Eighteen patients were Caucasian and two were Black. All cases corresponded to malignant neoplasm of the periorbital tissues. None of the cases was a primary tumor of the orbit or an intraocular tumor. Basal cell carcinoma of the eyelids was the main preoperative diagnosis $(45 \%)$. Other diagnoses responsible for the remaining $55 \%$ of the study population were: squamous cell carcinoma (25\%), Merkel cell carcinoma (15\%), sebaceous cell carcinoma (5\%), and malignant melanoma $(10 \%)$. The right eye was involved in $55 \%$ of the cases. The primary tumor affected the inner canthus $(30 \%)$ more often than the lateral canthus (11\%) and the superior eyelid (30\%) with higher frequency than the inferior lid (5\%), followed by the malar region that, in advanced cases, conditioned orbital and eye adnexal involvement (15\%), and both lids simultaneously (10\%). Mean follow-up time until IPO referral was 7.86 years (range 3 months to 20 years). Five patients $(25 \%)$ had already undergone previous surgery, outside the IPO, relating to the primary lesion which later progressed to exenteration. The preoperative diagnoses are summarized in Table 1.

At the beginning of the treatment at our institute, five patients $(25 \%)$ had severe visual loss in the affected eye and the remaining cases had severe extensive disease $(65 \%$ had an ulcer and $25 \%$ had a growing mass) with ocular and/or orbital structures (muscle and/or orbital fat) involvement.

All the patients were submitted to surgery less than a year after their first observation at our institute. The mean delay time between the diagnosis and the first visit to our center was 7.86 years. Almost half of the study sample (45\%) was evaluated in less than 12 months after diagnosis, although most of the patients (55\% of the sample) had a time delay in referral to IPO which ranged from 1 to 20 years, the latter value corresponding to one patient which fortunately represent, those, less frequent situations where exenteration was the final stage of several failed attempts to extirpate the tumor.

Table 1 Location and clinicopathologic classification of the primary tumors of 20 patients who underwent orbital exenteration at the Portuguese Oncology Institute of Porto

\begin{tabular}{|c|c|c|c|c|c|c|c|c|c|c|c|c|}
\hline \multirow[t]{2}{*}{ Location } & \multicolumn{2}{|c|}{$\begin{array}{l}\text { Basal cell carcinoma } \\
\text { (no of patients) }\end{array}$} & \multicolumn{2}{|c|}{$\begin{array}{l}\text { Squamous cell } \\
\text { carcinoma } \\
\text { (no of patients) }\end{array}$} & \multicolumn{2}{|c|}{$\begin{array}{l}\text { Malignant melanoma } \\
\text { (no of patients) }\end{array}$} & \multicolumn{2}{|c|}{$\begin{array}{l}\text { Sebaceous cell } \\
\text { carcinoma } \\
\text { (no of patients) }\end{array}$} & \multicolumn{2}{|c|}{$\begin{array}{l}\text { Merkel cell carcinoma } \\
\text { (no of patients) }\end{array}$} & \multicolumn{2}{|c|}{$\begin{array}{l}\text { Total } \\
\text { (no of patients) }\end{array}$} \\
\hline & Right (R) & Left (L) & $\mathrm{R}$ & $\mathrm{L}$ & $\mathrm{R}$ & $\mathrm{L}$ & $\mathrm{R}$ & $\mathrm{L}$ & $\mathrm{R}$ & $\mathrm{L}$ & $\mathrm{R}$ & $\mathrm{L}$ \\
\hline Inner canthus & 4 & 2 & - & - & - & - & - & - & - & - & 4 & 2 \\
\hline Lower eye lid & - & 1 & - & - & - & - & - & - & - & - & - & 1 \\
\hline Both eyelids & - & - & 1 & 1 & - & - & - & - & - & - & 1 & 1 \\
\hline Lateral canthus & 1 & - & 1 & - & - & - & - & - & - & - & 2 & - \\
\hline Malar skin & - & - & 1 & 1 & 1 & - & - & - & - & - & 2 & 1 \\
\hline Upper eyelid & 1 & - & - & - & - & 1 & - & 1 & 1 & 2 & 2 & 4 \\
\hline Total (no of patients) & 9 & & 5 & & 2 & & 1 & & 3 & & $\begin{array}{l}11 \\
20\end{array}$ & 9 \\
\hline
\end{tabular}


Oncological surgery and reconstruction

All patients underwent subperiosteal removal of the eye and all orbit contents (11 right and 9 left total exenterations). Total orbit exenteration was performed in cases of local invasive tumors when complete excision of the tumor with free margins was only possible with removal of the eye and all orbital contents in an attempt to achieve cure.

\section{Surgical technique}

Adequate wide margins were the main concern, yet with preservation of as much normal periocular soft tissue as possible. When it was necessary, adjacent areas of the medial canthus, temple, or forehead were included in the excision site. The skin was incised along mark and dissection was carried down through the periorbital to expose the orbital rim. The periorbital lining was mobilized along all orbital walls toward the orbital apex. When necessary, a frozen section was used to verify that the margins or resection are free and clear of neoplasm. The orbital bone was inspected for suspect bone pitting or other signs of bone erosion or destruction.

Complete histological excision was achieved in 16 patients $(80 \%)$. In four cases, with documented positive margins for malignant tumor, adjuvant therapy, in the form of orbit radiation was given.

The raw orbital cavity was repaired with a temporal muscular flap in all cases. The muscle flap was subsequently covered with one of three reconstructive options: full-thickness skin graft in five patients $(25 \%)$, bilaterally pedicle V-Y advancement flap in 2 patients (10\%), and a fasciocutaneous island flap of the retroauricular region in 13 patients $(65 \%)$.

The bilaterally pedicle V-Y advancement flap was chosen in order to possibly preserve at least part of the upper eyelid which was used to cover the upper half of the temporal muscle flap. The advancement flap was utilized for the lower half of the raw area and the wound was closed without tension. Because this flap has a reliable vascular basis of two subcutaneous pedicles that supply blood to the skin island through subdermal plexus lateral bridges, it was possible to cover a significant defect. This would not have been possible with a conventional V-Y flap [13] (Fig. 1).

The retroauricular fasciocutaneous island flap was successfully performed in 13 patients (65\% of the sample), there was anastomosis of the frontal branch of the temporal superficial artery to the corresponding vessel of the opposite side as well as with the ipsilateral supraorbital and supratrochlear arteries. The arc of rotation associated with the length of the pedicle allows its transfer in a one-stage procedure [14].

Complications occurred in $20 \%$ of the cases (four patients). In two cases (one with bilaterally pedicle V-Y advancement flap reconstruction and the other with a retroauricular fasciocutaneous island flap), infection and marginal tissue necrosis were reported and the treatment was local antibiotics and debridement. Later on, one of the patients developed a sinus fistula and underwent another surgical intervention (a cervicofacial flap was performed) with complete success. Two patients, one with a $\mathrm{CBC}$ and the other with a SCC had local recurrences and underwent local radiotherapy.

There is no report of systemic complications related to this surgical procedure; however, most of the patients were elderly and have significant co-morbidities.

The mean duration of hospital stay was 10 days. Most of the patients $(60 \%)$ had a short stay of 4 to 5 days. Longer periods of hospitalization were necessary for those four patients with the complications reported and for the other three patients with significant co-morbidities that required therapy unrelated to the periorbital tumor. Mean follow up was 2.28 years (range of 0.2 to 5 years)

\section{Discussion}

The most common indication for exenteration surgery is the treatment of epithelial malignancy together with orbital invasion [1-6]. To deal with this, periorbital tissues are usually excised to completely remove all potentially involved tissues. Although a highly disfiguring surgery, exenteration may provide a cure in cases of local invasive epithelial tumors such as basal and squamous cell carcinomas, this was clearly stated by Simon et al. in their study where they confirmed that when appropriate negative

Fig. 1 Socket reconstruction with temporal muscular flap and bilaterally pedicle V-Y advancement flap (13 months of follow up)

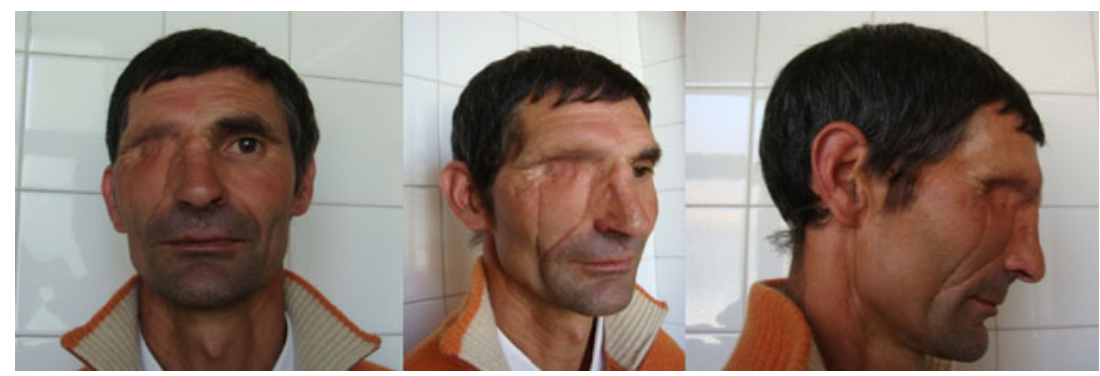


margins are assured, there rate is a low recurrence rate (7$10 \%$ ) and a low tumor-related death [1]. Our series corroborates other published data where the main indication for exenteration was the treatment of cutaneous malignancy that was not amenable to local excision without compromising the eye. Irradiation, or other topical treatment modalities were also used [1-6].

As in most published work, in our experience, the majority of our patients are elderly Caucasian males [4]. Regarding tumor location, other studies indicate that $80 \%$ to $90 \%$ of all malignant tumors situated on the eyelids affect primarily the inner canthus which corroborates with our results [5]. According to this study series, the upper lid and the inner canthus were most affected.

Basal cell carcinoma was responsible for $45 \%$ of exenteration in our sample. Similar results were reported in several other studies such as the work by Nassab et al. in which basal cell carcinoma was the main surgical indication accounting for $53 \%$ of all tumors [5].

The second most common malignancy demanding exenteration in our series was squamous cell carcinoma (which accounted for $25 \%$ of the cases). Several published works corroborate our data [4-6] indicating that squamous cell carcinoma is the second most common malignant neoplasm of the eyelid (nearly $5 \%$ to $10 \%$ of all eyelid malignancies). However, other reports describe squamous cell as the most prevalent tumor for which exenteration was performed [1, 2]. That might be interpreted as being a consequence of early diagnosis and treatment of basal cell carcinoma, most likely because of a high index of suspicion in the early stages and the introduction of Mohs micrographic surgery. On the other hand, because of perineural invasion, squamous cell carcinomas might require more extensive surgery.

It is estimated that cutaneous malignant melanoma of the eyelid accounts for about $1 \%$ of all eyelid malignancies [5]. In our series, it represented the third largest indication for surgery ( $10 \%$ of the cases).

Sebaceous gland carcinoma, although relatively rare, is described as being responsible for about $10 \%$ of all eyelid malignancies with a propensity for the upper eyelid, female sex, and often affecting adults in the sixth to seventh decades $[5,7,15]$. We had one male patient with inferior eyelid involvement in the seventh decade of life, which accounted for $5 \%$ of our cases.

Merkel cell carcinoma of the eyelid is known to be a rare neoplasm with a predilection for the upper eyelid. In our sample, we had one case of upper eyelid involvement [16, 17].

All patients underwent immediate local flap reconstruction with temporalis muscle. There are several different reconstructive options available. Spontaneous granulation and skin grafting is the simplest. The main disadvantage of the first technique is the prolonged healing time with inherent risk of infection and the socket provided has a thin and fragile covering (admitting a complete take of the graft, which not always happens) vulnerable to trauma, with a unsatisfactory appearance [18]. The ease of clinical examination and recurrence surveillance is repeatedly pointed as an important and unique advantage of this technique. However, clinical monitoring can no longer be a true concern in view of all the currently available technology (MRI, CT scan) $[4,5]$. Thus, covering the socket with a temporalis muscle flap is, in what concerns tumor surveillance, as safe as skin grafting [19], with other additional advantages, such as higher resistance to infection and radiation therapy. In our series, all patients underwent immediate reconstruction and we achieved stable results without major postoperative complications. The coverage of the muscle flap with one of the three options described improved the appearance and enhanced the stability and resistance of the reconstructive procedure. Traditionally, the choice of this and other local flaps as reconstructive options is faced with apprehension and reservation when concerning elderly patients. Our results show this is a safe and reliable option without additional or increased complications. Furthermore, the higher resistance of this type of reconstruction to adjuvant therapies such as radiotherapy is well known, which assumes a great impact is what concerns complicated patients with other comorbidities $[2-5,10]$.

This work reinforces the importance of early diagnosis, appropriate treatment as well as careful and regular monitoring to detect any recurrences and act accordingly. Temporal flap muscle and cutaneous covering with skin grafts or local flaps are safe and reliable options with longterm stability, higher resistance to complementary treatments, and reasonable aesthetic results with minimal associated donor site morbidity.

Disclosure None of the authors has a financial interest to declare in relation to the content of this article.

\section{References}

1. Simon GJB, Schwarcz RM, Douglas R et al (2005) Orbital exenteration: one size does not fit all. Am J Ophthalmol 139:11-17

2. Tacla M, Paves L et al (2006) Exentração: estudo retrospectivo. Arq Bras Oftalmol 9(5):679-682

3. Rathbun J, Beard C, Quickert MH (1971) Evaluation of 48 cases of orbital exenteration. Am J Ophthalmol 30:191-199

4. Mouriaux F, Barraco P et al (2001) L'exentération orbitaire. J Fr Ophthalmol 24(8):865-874

5. Nassab R, Thomas S (2007) Orbital exenteration for advanced periorbital skin cancers: 20 years experience. J Plast Reconstr Aesth Surg 60:1103-1109

6. Cook BE Jr, Bartley GB (2001) Treatment options and future prospects for the management of eyelid malignancies: an evidence-based update. Ophthalmology 108(11):2088-2098 
7. Shields JA, Demirci H et al (2004) Sebaceous carcinoma of the eyelids: personal experience with 60 cases. Ophthalmology 111 (12):2151-2157

8. Frezzotti R, Bonanni R et al (1992) Radical orbital resections. Adv Ophthalmic Plast Reconstr Surg 9:175-192

9. Cuesta-Gil M, Concejo C et al (2004) Repair of large orbitocutaneous defects by combining two classical flaps. J Craniomaxillofac Surg 32:21-27

10. Menon N, Girotto JA, Goldberg NH, Silverman RP (2003) Orbital reconstruction after exenteration: use of a transorbital temporal muscle flap. Ann Plast Surg 50:38-42

11. Wax M, Burkey BB et al (2003) The role of free tissue transfer in the reconstruction of massive neglected skin cancers of the head and neck. Arch Facial Plast Surg 5:479482

12. Uusitalo M, Ibarra M, Fulton L et al (2001) Reconstruction with rectus abdominis myocutaneous free flap after orbital exenteration in children. Arch Ophthalmol 119:1705-1709
13. Pontes L, Ribeiro M et al (2002) The new bilaterally pedicled V-Y advancement flap for face reconstruction. Plast Reconstr Surg 109 (6): $1870-1874$

14. Pinho C, Choupina $M$ et al (2003) A new retroauricular flap for facial reconstruction. Br J Plast Surg 56(6):599-602

15. Snow SN, Larson PO et al (2002) Sebaceous carcinoma of the eyelids treated by Mohs micrographic surgery: report of nine cases with review of the literature. Dermatol Surg 28(7):623-631

16. Pathai S, Barlow R, Williams G, Oliver J (2005) Mohs' micrographic surgery for Merkel cell carcinomas of the eyelid. Orbit 24:273-275

17. Bleyen I, Wong J et al (2010) Merkel cell carcinoma of the eyelid: a report of 2 cases. Can J Ophthalmol 45(1):85-86

18. Putterman AM (1986) Orbital exenteration with spontaneous granulation. Arch Ophthalmol 104(1):139-140

19. Mauriello JA Jr, Han KH et al (1985) Use of autogenous splitthickness dermal graft for reconstruction of the lining of the exenterated orbit. Am J Ophthalmol 15;100(3):465-467 